\title{
A set of microRNAs mediate direct conversion of human umbilical cord lining-derived mesenchymal stem cells into hepatocytes
}

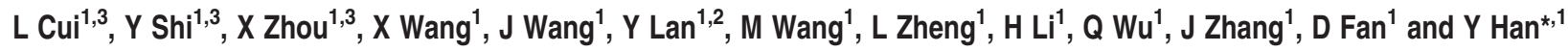

In a previous study, we elucidated the specific microRNA (miRNA) profile of hepatic differentiation. In this study, we aimed to clarify the instructive role of six overexpressed miRNAs (miR-1246, miR-1290, miR-148a, miR-30a, miR-424 and miR-542-5p) during hepatic differentiation of human umbilical cord lining-derived mesenchymal stem cells (hMSCs) and to test whether overexpression of any of these miRNAs is sufficient to induce differentiation of the hMSCs into hepatocyte-like cells. Before hepatic differentiation, hMSCs were infected with a lentivirus containing a miRNA inhibitor sequence. We found that downregulation of any one of the six hepatic differentiation-specific miRNAs can inhibit HGF-induced hepatic differentiation including albumin expression and LDL uptake. Although overexpression of any one of the six miRNAs alone or liver-enriched miR-122 cannot initiate hepatic differentiation, ectopic overexpression of seven miRNAs (miR-1246, miR-1290, miR-148a, miR30a, miR-424, miR-542-5p and miR-122) together can stimulate hMSC conversion into functionally mature induced hepatocytes (iHep). Additionally, after transplantation of the iHep cells into mice with CCL4-induced liver injury, we found that iHep not only can improve liver function but it also can restore injured livers. The findings from this study indicate that miRNAs have the capability of directly converting hMSCs to a hepatocyte phenotype in vitro.

Cell Death and Disease (2013) 4, e918; doi:10.1038/cddis.2013.429; published online 14 November 2013

Subject Category: Experimental Medicine

The goal of regenerative medicine is to replace the cells that are damaged or lost as we age, suffer disease or are exposed to environmental insults. For patients with liver disease, the ideal regeneration process is cell transplantation of hepatocytes generated by transdifferentiation to supplement or replace hepatocyte function. Rapid advances in the field of transdifferentiation have been made, particularly in hepatic transdifferentiation. Mouse tail-tip fibroblasts can be directly induced to functional hepatocyte-like cells by transduction of Gata4, Hnf1alpha and Foxa3 and inactivation of p19 (Arf); the induced hepatocytes show typical epithelial morphology, express hepatic genes and acquire hepatocyte functions. Notably, transplanted induced hepatocytes repopulate the livers of fumarylacetoacetate-hydrolase-deficient (Fah $(-/-))$ mice and rescue almost half of the Fah $(-/-)$ mice from death by restoring liver function. ${ }^{1}$ Simultaneously, another research group demonstrated that expression of the transcription factor Hnf4alpha in combination with Foxa1, Foxa2 or Foxa3 can convert mouse embryonic and adult fibroblasts into cells that closely resemble hepatocytes in vitro. The induced hepatocyte-like cells have multiple hepatocyte-specific features and can also reconstitute damaged hepatic tissues after transplantation. ${ }^{2}$ A surprising observation is that transcription factors are not the only molecules that can promote cell transdifferentiation; miRNAs can promote cell transdifferentiation as well. ${ }^{3-5}$ Whether miRNA can mediate hepatic transdifferentiation is still unknown.

In the previous study, we tested the miRNA expression profile of the HGF-induced hepatic differentiation model using miRNA microarray at seven time points. A total of 61 miRNAs among 1205 human and 144 human viral miRNAs displayed consistent changes and were altered at least twofold between hUC-MSCs and hepatic differentiated hUC-MSCs. Then, 25 miRNAs were selected based on fold changes and expression level for further qRT-PCR analyses. By comparing this miRNA profile between osteogenic differentiated cells and hepatocyte differentiated cells, we found that miR-1246, miR-1290, miR-148a, miR-30a, miR-424 and miR-542-5p not only were consistently overexpressed during hepatic differentiation of

\footnotetext{
${ }^{1}$ State Key Laboratory of Cancer Biology, Xijing Hospital of Digestive Diseases, The Fourth Military Medical University, Xi'an, Shaanxi Province, China and ${ }^{2}$ Department of Gastroenterology, Yan'an University Affiliated Hospital, Yan'an, Shaanxi Province, China

${ }^{*}$ Corresponding author: Y Han, State Key Laboratory of Cancer Biology, Xijing Hospital of Digestive Diseases, The Fourth Military Medical University, 127 West Chang-le Road, Xi'an 710032, Shaanxi Province, China. Tel: +86 29 84771515; Fax: +86 29 82539041; E-mail: hanying @fmmu.edu.cn

${ }^{3}$ These authors contributed equally to this work.

Keywords: miRNA; mesenchymal stem cells; hepatic differentiation; hepatocytes

Abbreviations: AFP, $\alpha$-fetoprotein; ALB, albumin; BUN, blood urea nitrogen; cDNA, complementary deoxyribonucleic acid; CCL4, carbon tetrachloride; CK, cytokeratin; CYP, cytochrome P-450; eGFP, enhanced green fluorescent protein; EpCAM, epithelial cell adhesion molecule; Fah, fumarylacetoacetate-hydrolase; Foxa3, forkhead box a3; GATA4, GATA-binding protein 4; HGF, hepatocyte growth factor; HNF, hepatic nuclear factor; hUC, human umbilical cord; ICG, cardiogreen; iHEP, induced hepatocyte; LDL, low-density lipoprotein; LEV, lentivirus; miRNA, microRNA; MSC, mesenchymal stem cell; MOI, multiplicity of infection; PAS, periodic acid-Schiff; PDX1, pancreatic and duodenal homeobox 1; RNA, ribonucleic acid

Received 07.5.13; revised 25.8.13; accepted 12.9.13; Edited by Y Shi
} 
hMSCs either by microarray or qRT-PCR analysis but also were hMSC hepatic differentiation specifically. ${ }^{6}$ In this study, we aimed to clarify the instructive role of the six overexpressed miRNAs during hepatic differentiation of hMSCs and to test whether overexpression of any of these miRNAs alone is sufficient to induce differentiation of hMSCs into hepatocyte-like cells.

\section{Results}

Downregulation of any one of the six hepatic differentiationspecific miRNAs can inhibit HGF-induced hepatic differentiation. To clarify the role of the six miRNAs that are overexpressed during hMSC hepatic differentiation, we infected hMSCs with miRNA-RNAi-Lev before HGF-induced hepatic differentiation. After induction with HGF for 6 days, the albumin mRNA expression of hMSCs and hMSCs infected with miRNA-control-Lev was upregulated 4.6-fold and 3.4-fold, respectively. Whereas hMSCs infected with miR-1246-RNAi-Lev, miR-1290-RNAi-Lev, miR-148a-RNAiLev, miR-30a-RNAi-Lev, miR-424-RNAi-Lev or miR-542-5pRNAi-Lev did not overexpress albumin (Figure 1b). Moreover, after HGF-induction for 12 days, hMSCs successfully infected with miRNA-control-Lev (indicated by green fluorescence) were able to take up Ac-Dil-LDL (indicated by red fluorescence); however, hMSCs successfully infected with miR-1246-RNAi-Lev, miR-1290-RNAi-Lev, miR-148a-RNAi-Lev, miR-30a-RNAi-Lev, miR-424-RNAiLev, miR-542-5p-RNAi-Lev (indicated by green fluorescence) could not take up Ac-Dil-LDL (indicated by the absence of red fluorescence), and only several hMSCs without miRNA-RNAi-Lev infection were able to take up Ac-Dil-LDL (indicated by red fluorescence) (Figure 1c). None of the six miRNA-RNAi-Levs inhibited HNF4A expression (Figure 1b). Additionally, miR-1246-RNAi-Lev, miR1290-RNAi-Lev, miR-148a-RNAi-Lev, miR-30a-RNAi-Lev,

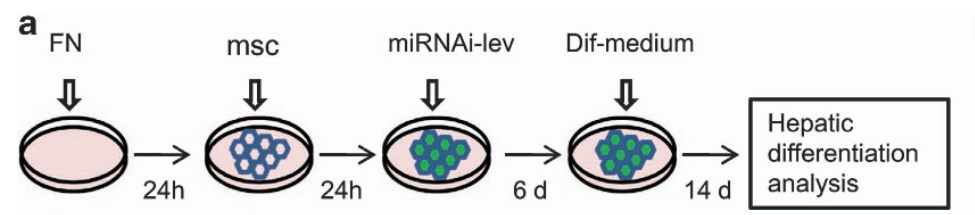

b
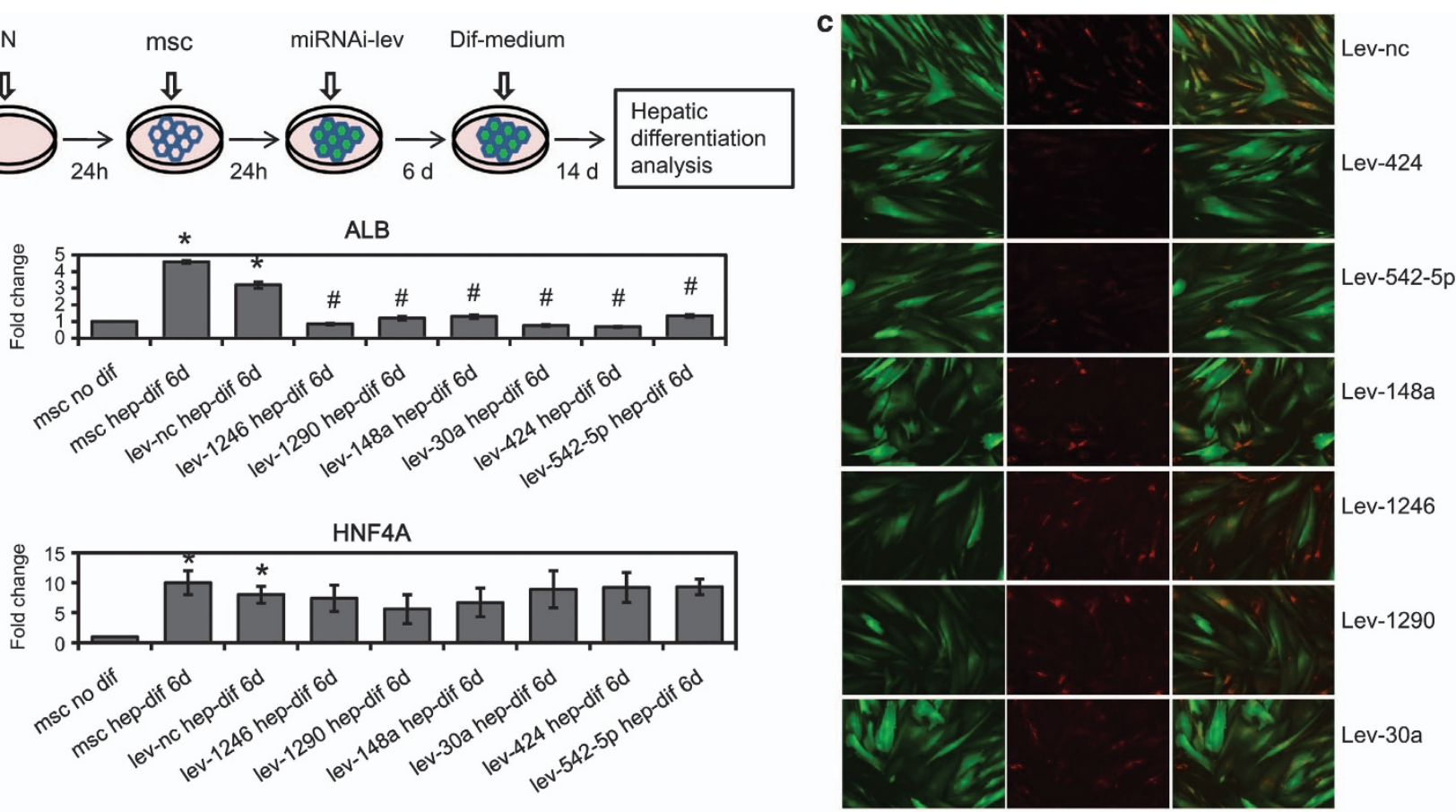

d

ALB
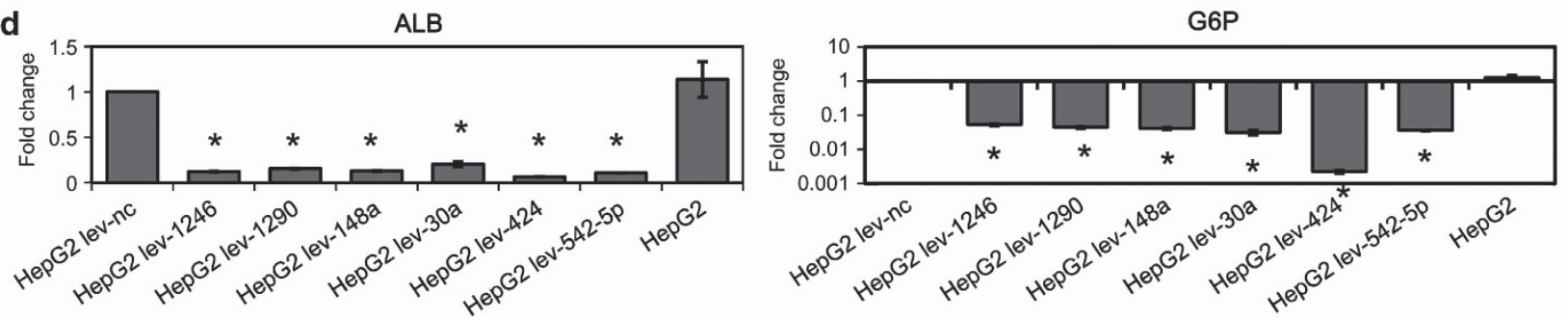

Figure 1 Downregulation of miRNAs can inhibit HGF-induced hepatic differentiation. (a) Experimental design of the effects of miRNA on hepatic differentiation. hMSCs seeded on FN-pretreated culture plates were infected with lentiviruses expressing miRNA inhibitor sequences. The cultures were changed to hepatic differentiation medium 6 days after infection. (b and $\mathbf{d}$ ) Hepatocyte-specific gene expression analyzed by qRT-PCR. Hepatocyte-specific gene expression was normalized to GAPDH expression, and the results are expressed relative to a value of one in the control hMSCs. The data represent means \pm standard deviation $(n=3)$. (b) Albumin and HNF4A expression after hepatic differentiated hMSCs were infected with miRNAi-Lev. ("compared with msc, $P<0.05$ by Student's $t$-test; \#compared with lev-nc, $P<0.05$ by Student's $t$-test). (d) Albumin and G6P expression in HepG2 cells infected with miRNAi-Lev. ("'compared with HepG2 lev-nc, $P<0.05$ by Student's $t$-test) (c) Analysis of the LDL uptake ability of hepatic differentiated hUC-MSCs infected with miRNAi-Lev (green: EGFP; red: Dil-LDL) 
miR-424-RNAi-Lev and miR-542-5p-RNAi-Lev can downregulate $\mathrm{ALB}$ and $\mathrm{G} 6 \mathrm{P}$ expression in HepG2 cells (Figure 1d). These results indicate that miR-1246, miR1290, miR-148a, miR-30a, miR-424 and miR-542-5p may have an essential role in hepatic differentiation of hMSCs.

Overexpression of seven miRNAs together can stimulate hMSCs to possess the characterization of hepatocytes. Silencing any one of the six hepatic differentiation-related miRNAs can inhibit the HGF-induced ALB expression and LDL uptake during hepatic differentiation of hMSCs. To clarify the effects of overexpression of the six miRNAs on hMSCs, we synthesized the miRNA mimics and used the liver-enriched miRNA miR-122 as a control. To confirm that the synthesized miRNA mimics can effectively increase the relative expression level of miRNA in hMSCs, we tested the miRNA expression of hMSCs at 6 days post transfection. We found that the miRNA mimics miR-122, miR-1246, miR-1290, miR-148a, miR-30a, miR-424 and miR-542-5p increased the relative expression of their respective miRNAs 18163-, 2-, 8-, 87-, 11-, 117- and 16542 -fold, respectively (Figure 2a). The fold change difference may be caused by the different expression level of the miRNA in hMSC. This overexpression was maintained for $\sim 12$ days. However, overexpression of any of the seven miRNAs alone cannot stimulate hMSCs to express the hepatocyte marker gene ALB (Figure $2 b$ ). We combined the seven miRNA mimics and co-transfected them into hMSCs. Co-transfection increased the relative expression of all seven miRNAs simultaneously (Figure 2c). Interestingly, after cotransfection of the seven miRNA mimics for 6 days, the hMSCs overexpressed ALB (Figure 2d). Moreover, the combination of the seven miRNAs can promote the conversion of hMSCs from a fibroblast-like morphology to an epithelial morphology (Figure 2e and Supplementary Figure 1C).

Co-transfection with a combination of seven miRNAs can convert hMSCs into mature functional hepatocytes in vitro. Human liver multipotent stem/progenitor cells can give rise to hepatocytes, cholangiocytes and
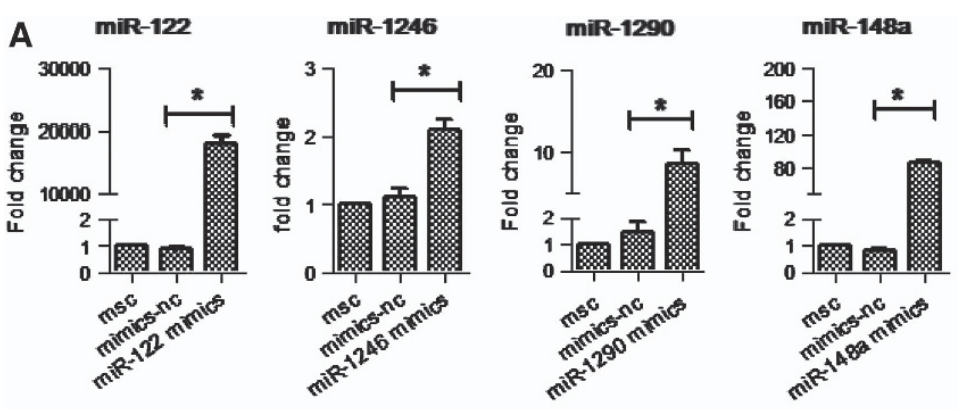

B

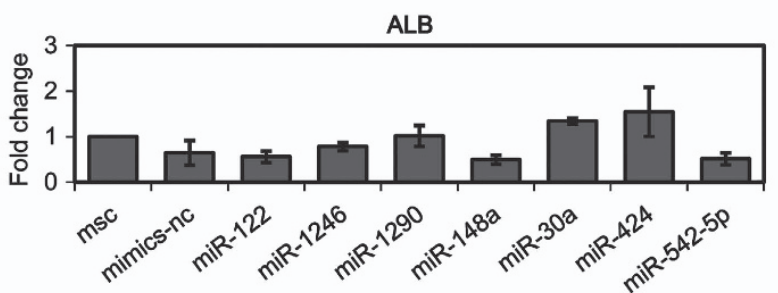

C

miRNA expression

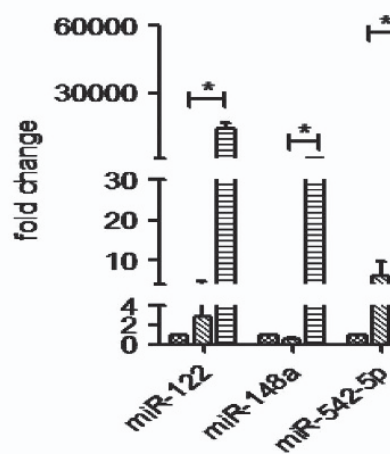

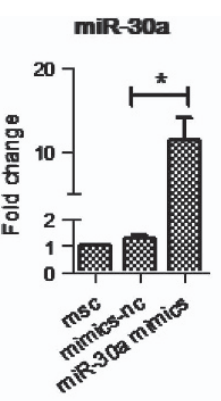
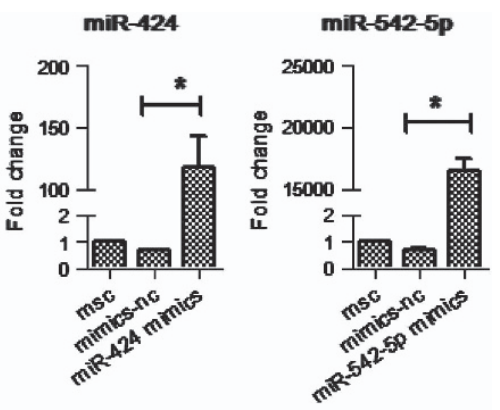

D

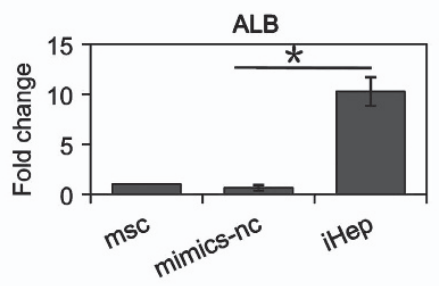

E

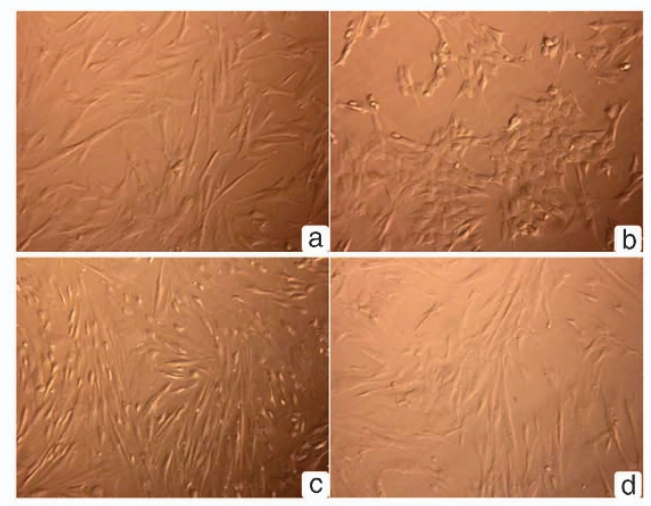

Figure 2 Effects of single miRNAs or combinations of miRNAs on hMSCs. (A) The relative expression of miRNA in hMSCs transfected with single miRNA mimics analyzed by qRT-PCR at day 6. (B) Albumin expression in hMSCs transfected with a single miRNA mimic for 6 days was analyzed by qRT-PCR. (C) Ectopic miRNA expression in hMSCs transfected with seven miRNA mimics in combination was analyzed by qRT-PCR at day 6. (D) Albumin expression in hMSCs transfected with seven-miRNA mimics for 6 days was analyzed by qRT-PCR. (E) Morphology of hMSCs before and after miRNA transfection for 6 days. (a) hMSC; (b) hMSC treated with seven-miRNA; (c) hMSC treated with mimic-nc; (d) hMSC treated with hepatic differentiation medium. The data represent means \pm standard deviation $(n=3)(\mathbf{A}-\mathbf{D})$. ${ }^{*}$ compared with mimics-nc, $P<0.05$ by Student's $t$-test, $(\mathbf{A}-\mathbf{D})$ 
pancreatic islets. To confirm that the seven-miRNA combination converts hMSCs into hepatocytes but not into hepatic progenitor cells, pancreatic islets or cholangiocytes, we analyzed the expression of marker genes in hMSCs cotransfected with the seven-miRNA combination. qRT-PCR results showed that the seven-miRNA combination can stimulate hMSCs to express hepatocyte-specific genes, including the early genes HNF4A, AFP and albumin (which were increased 8.2-, 2.1- and 10.3-fold, respectively), the intermediate gene transferrin (which was increased 5.2-fold) and the late gene CYP3A4 (which was increased 4.6-fold). However, the seven-miRNA combination cannot stimulate the expression by hMSCs of the pancreatic islet marker gene PDX1, the cholangiocyte marker gene CK7 or the liver progenitor cell marker gene EpCAM (Figure 3a). We also analyzed whether hMSC mediated by seven-miRNA combination could induce hepatocellular carcinoma in vivo. We found that transplant tumor was not formed in nude mice after cells were vaccinated into subcutaneous tissue of nude mice (data not shown). To further confirm that the cells mediated by the seven-miRNA combination can successfully express hepatocyte marker genes, we analyzed the protein levels of HNF4A, ALB and CYP3A4 by western blot. The western blot results were consistent with the qRT-PCR results (Figure 3b and Supplementary Figure $1 \mathrm{C}$ ).

To clarify whether the induced hepatocytes (iHeps) mediated by the seven-miRNA combination have hepatocyte function in vitro, we examined their glycogen-storing ability, ICG and LDL uptake ability, urea production ability and albumin-positive cells percentage. The ability of iHeps to produce urea was evaluated by exposing the cells to $10 \mathrm{mmol} / \mathrm{l}$ ammonium chloride for $24 \mathrm{~h}$. The urea production ability of iHeps increased significantly after 6 days of transfection with the seven-miRNA combination (Figure 3c). After induction for 6 days, PAS staining demonstrated that $>70 \%$ cells stored glycogen compared with the undifferentiated hUC-MSCs
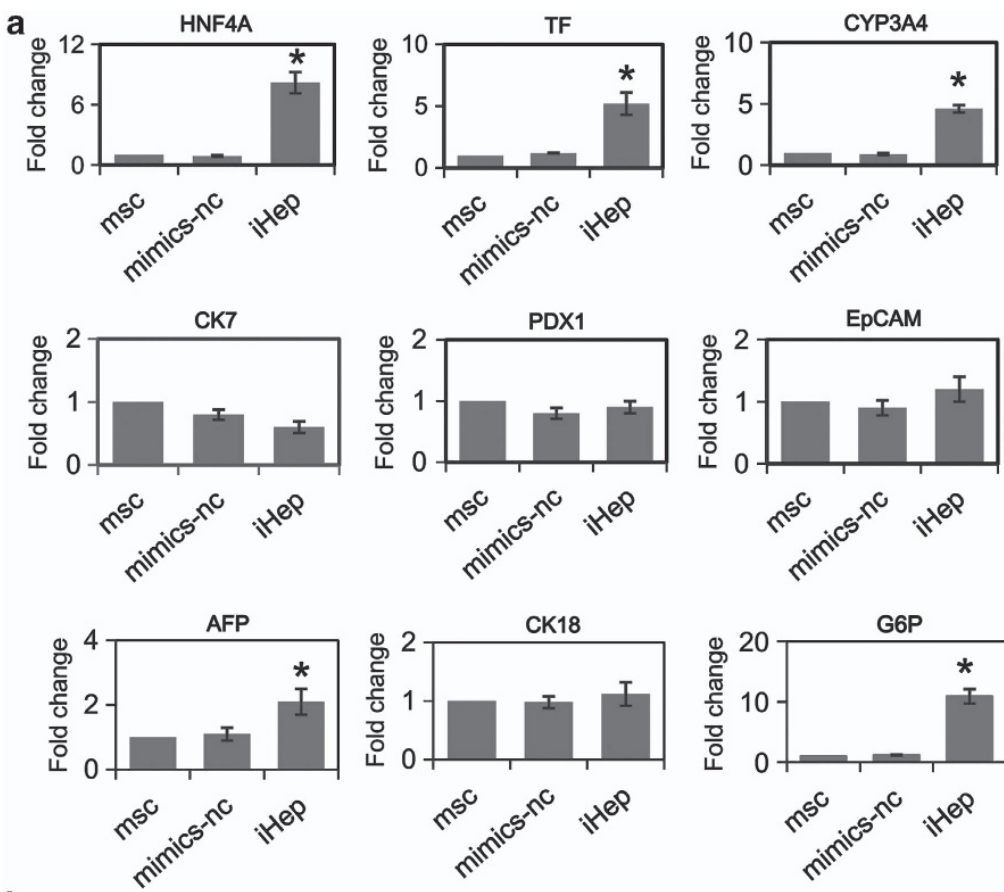

b

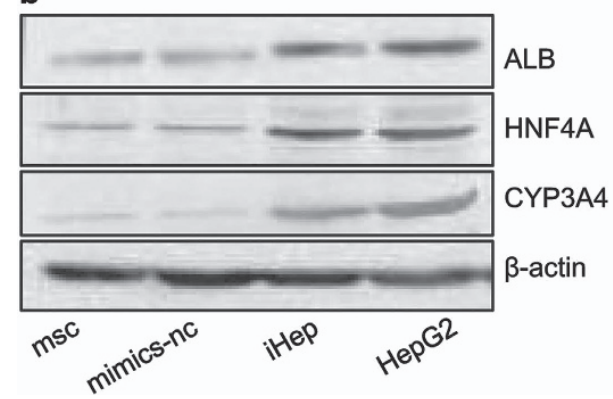

。
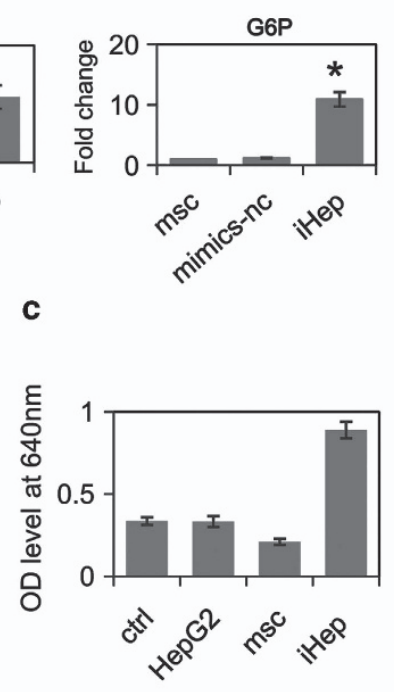
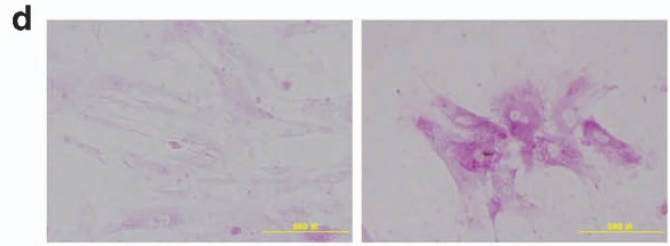

e

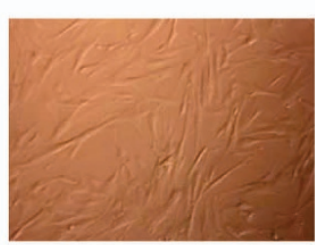

mimics-nc

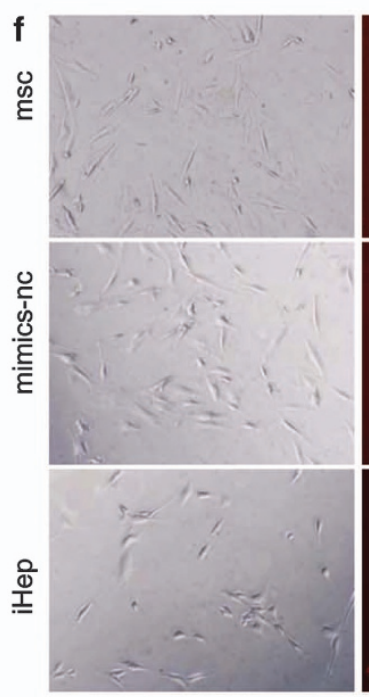

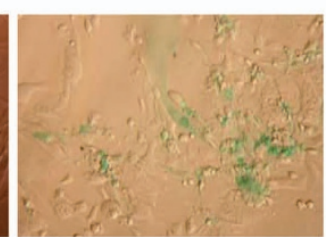

$\mathrm{iHep}$

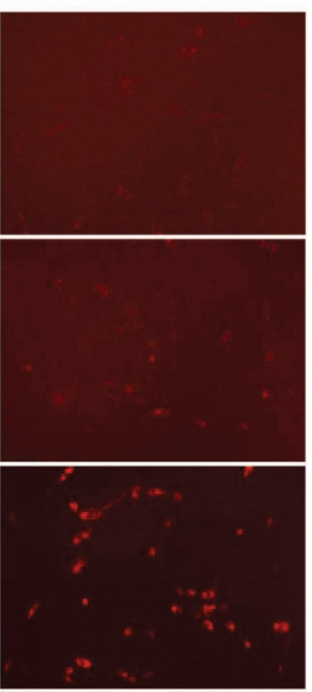

Figure 3 The seven-miRNA combination can convert hMSCs into mature functional hepatocytes in vitro. Hepatic function analysis of hMSCs before and after sevenmiRNA co-transfection for 6 days. (a) Hepatocyte-specific gene expression analyzed by qRT-PCR. The data represent means \pm standard deviation ( $n=3$ ). ${ }^{*}$ Compared with mimics-nc, $P<0.05$ by Student's t-test. (b) Hepatocyte-specific gene expression analyzed by western blot. (c) Analysis of the BUN synthetic ability of hMSC mediated by the seven-miRNA combination. (d) PAS staining of hMSC mediated by the seven-miRNA combination. (e) ICG uptake analysis of hMSC mediated by the seven-miRNA combination. (f) Analysis of the LDL uptake ability of hMSC mediated by the seven-miRNA combination 
(Figure 3d and Supplementary Figure 1D). Moreover, approximately half of the iHeps could take up ICG (Figure 3e) and almost all of the iHeps could take up LDL (Figure $3 f$ and Supplementary Figure 1D). Immunofluorescence results demonstrated that $>90 \%$ cells were albuminpositive (Supplementary Figure 1A and 1D). These results indicated that hepatocyte induction mediated by the sevenmiRNA combination can exert hepatocyte function in vitro.

iHep transplantation rescues CCL4-induced liver injury mice. Hepatocyte transplantation can reverse acute liver injury. Therefore, we examined whether human iHep cells could reconstitute hepatic tissues as hepatocytes in the livers of a CCL4-induced acute liver injury model. Mice treated with CCL4 for 4 weeks exhibited weight loss and loss of appetite. The histological evaluation of the CCL4-injured mice demonstrated that compared with normal mice (Figure $4 \mathrm{Ci}$ ), ballooning and necrosis of hepatocytes, infiltration of inflammatory markers and liver fibrosis increased significantly (Figure 4Cii). Moreover, the liver function of CCL4-injured mice decreased as indicated by an increase in alanine transaminase (ALT) (from 21.3 $\pm 3.4 \mathrm{UI} / \mathrm{l}$ to $243 \pm 13 \mathrm{UI} / \mathrm{I}$ ) and aspartate aminotransferase (AST) (from $108 \pm 10 \mathrm{UI} / \mathrm{l}$ to $486 \pm 20 \mathrm{UI} / \mathrm{l})$ and a decrease in serum albumin (from $23 \pm 1.4 \mathrm{~g} / \mathrm{l}$ to $15.7 \pm 0.6 \mathrm{~g} / \mathrm{l}$ ). We treated the liver-injured mice with saline, negative cells, hMSC or iHep cells injection. Interestingly, 1 day after iHep cell transplantation, the serum albumin level was significantly increased (from $15.7 \pm 0.6 \mathrm{~g} / \mathrm{l}$ to $24.4 \pm 0.45 \mathrm{~g} / \mathrm{l}$ ) and a normal level was maintained during the observation period. Conversely, the serum albumin level in mice treated with hMSCs showed gradual improvement from days $1-14$ (from $15.8 \pm 0.5 \mathrm{~g} / \mathrm{l}$ to $19.8 \pm 0.28 \mathrm{~g} / \mathrm{l})$, and the serum albumin in mice treated with saline or negative cell was maintained at a low level (Figure 4b). Both iHep and hMSC cells repaired the injured liver architecture at 2 weeks post cell transplantation (Figure 4c) and exhibited decreased ALT and AST levels (Figure 4b).

a
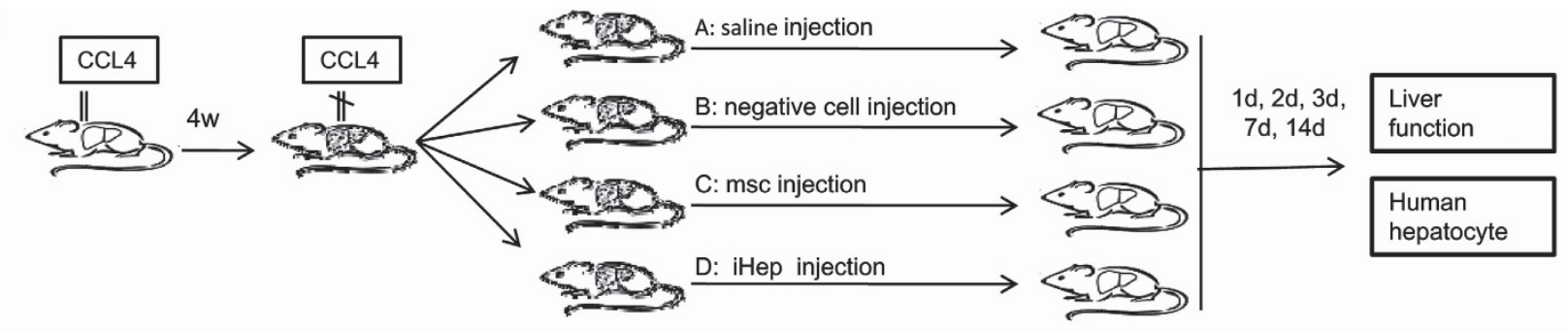

b
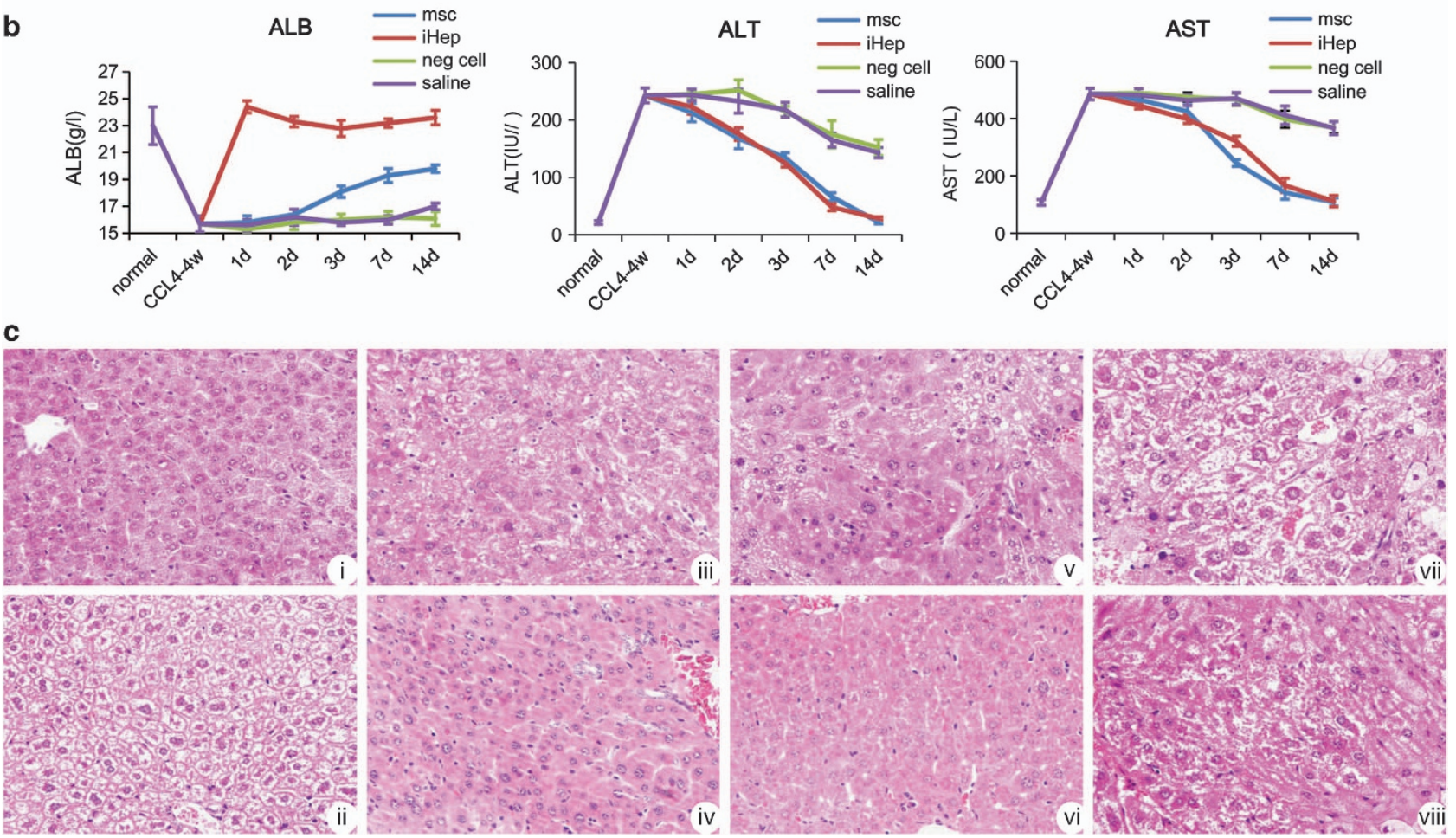

Figure 4 iHep transplantation restores liver function of CCL4-induced liver-injured mice. (a) Schematic outline of iHep cell transplantation into the livers of CCL4-injured mice. (b) Serum ALB, ALT and AST levels after cell transplantation. (c) H\&E staining of liver tissue from mice exposed to different treatments. i normal; ii: CCL4 treatment for 4 weeks; iii: hMSC transplantation for 7 days; iv: hMSC transplantation for 14 days; v: iHep transplantation for 7 days; vi: iHep transplantation for 14 days; vii: negative cell transplantation for 14 days; viii: saline injection for 14 days 
To further confirm that the decreased liver function was improved by transplantation of iHep cells, we traced the transplanted hMSC and iHep cells in liver sections from the mice using immuofluorescence. In the hMSC treatment group, the human-derived cells were mainly CD105-positive cells. However, in the iHep treatment group, the humanderived cells were mainly albumin-positive and CYP3A4positive cells. In the hMSC treatment group, the human CD105-positive MSCs in the liver gradually increased, reached the highest level on day 3 and then gradually decreased. Additionally, human albumin-positive and CYP3A4-positive hepatocytes began to increase at day 3 . In the iHep treatment group, the human albumin-positive and CYP3A4-positive hepatocytes in the liver gradually increased and reached the highest level on day 3 and that level was then stably maintained (Figure 5 and Supplementary Figure 2). More interestingly, human CD105-positive MSCs maintained a stable low level from days 1-14 (Figure 5). These results indicated that iHep cell transplantation can repair liver injury in mice.

\section{Discussion}

MicroRNAs (miRNA) comprise a group of non-coding small RNAs (17-25 nt) that are involved in post-transcriptional regulation and have been identified in various plants and animals. They have an important role in liver development. MiRNA-deficient mice exhibited progressive hepatocyte damage with elevated serum ALT and AST levels between 2 and 4 months of age. Furthermore, the liver mass and expression of cellular markers of both proliferation and apoptosis were shown to increase. ${ }^{7}$ Moreover, miRNAs control hepatocyte proliferation during liver regeneration ${ }^{8,9}$ and have a significant role in modulating proliferation and cell cycle progression genes after partial hepatectomy. ${ }^{10}$ In the previous study, we found that miR-1246, miR-1290, miR-148a, miR-30a, miR-424 and miR-542-5p were specifically overexpressed during hepatic differentiation of hMSCs. ${ }^{6}$ Thus far, studies on these miRNAs have been limited. MiR1246 takes part in DNA damage, ${ }^{11}$ regulates chloride transport ${ }^{12}$ and can be used as a circulating biomarker of malignant mammary epithelial cells. ${ }^{13}$ MiR-1290 impairs cytokinesis and affects the reprogramming of colon cancer cells. ${ }^{14}$ MiR-30a inhibits the epithelial-to-mesenchymal transition by targeting Snai1, ${ }^{15}$ and the miR-30 family is required for vertebrate hepatobiliary development. ${ }^{16} \mathrm{MiR}-148 \mathrm{a}$ is a promising candidate for an early, stable and sensitive biomarker of rejection and hepatic injury after liver transplantation. ${ }^{17}$ MiR-424 regulates human monocyte/macrophage differentiation. ${ }^{18}$ Studies on miR-542-5p mainly focused on its important role in tumorigenesis ${ }^{19,20}$ and its part in the cellular senescence program of human diploid fibroblasts. ${ }^{21}$ In this study, we found that these six miRNAs not only have key roles in the HGF-induced hepatic differentiation of hMSCs but also influence hepatic gene expression in the HepG2 cell line. Moreover, we found that although upregulation of any one of the six miRNAs alone or miR-122 in hMSCs cannot initiate hepatic differentiation, ectopic overexpression of seven miRNAs together can convert hMSCs into mature functional hepatocytes. MiR-122 is highly expressed in the liver where it constitutes $70 \%$ of the total miRNA pool. MiR-122 participates in cholesterol metabolism and hepatocellular carcinoma formation and has an important role in promoting hepatitis $\mathrm{C}$ virus replication. ${ }^{22}$ Overexpression of miR-122 enhances in vitro hepatic differentiation of fetal liver-derived stem/ progenitor cells, ${ }^{23}$ but whether miR-122 can initiate hepatic differentiation of hMSCs is still unknown. Here, we showed that ectopic overexpression of miR-122 alone cannot initiate hepatic differentiation of hMSCs, but when combined with miR-1246, miR-1290, miR-148a, miR-30a, miR-424 and miR542-5p, miR-122 can convert hMSCs into functional hepatocytes.

HMSCs are plastic. They not only possess osteogenic, chondrogenic and adipogenic differentiation potential but can also break through the limitation of their germ layer of origin and differentiate into hepatocyte-like cells under hepatogenic conditions. Until now, the mechanism of hepatic differentiation was unknown. In the past few years, much work has been done to understand the hepatogenic condition. The conventional and most frequently used hepatogenic medium is basic medium supplemented with growth factors including epidermal growth factor, bFGF, HGF, nicotinamide, oncostatin $M$, dexamethasone and ITS premix. ${ }^{24}$ In this study, we directly converted hMSCs into hepatocytes using a seven-miRNA combination. Compared with conventional methods, the seven-miRNA combination promoted hepatic differentiation of hMSCs more quickly and more efficiently. For example, after 6 days of induction, growth factors upregulated albumin expression approximately fourfold, whereas the sevenmiRNA combination upregulated albumin expression 10-fold. Furthermore, the uptake of Ac-Dil-LDL and the storage of glycogen by hMSCs were induced by the seven-miRNA combination after 6 days, whereas these hepatic functions were not observed in the conventional induction group until 14 days after induction. Additionally, the seven-miRNA combination can change the cell morphology from fibroblast-like to epithelial; this phenomenon was not observed in the conventional induction group. Therefore, compared with the conventional induction method, the seven-miRNA combination is a simpler, faster, more efficient method of hepatic induction. Recently, transcription factor-based cellular reprogramming has opened the door to the conversion of somatic cells ${ }^{1,2}$ or stem cells ${ }^{25,26}$ to hepatocytes; however, several limitations currently prohibit the use of this method in clinical settings, including the viral DNA delivery system and the exogenous overexpression of transcription factors. New strategies are therefore needed to ensure the safe and efficient production of hepatocytes. Accumulating evidence now implicates miRNAs as probable candidates for cellular reprogramming. These transient, non-coding small RNAs are fully or partially complementary to one or more mRNA molecules and induce the silencing of targeted genes without integration into the host genome.

Both MSC and hepatocyte transplantation can improve liver function in patients or in animals with liver disease. Studies demonstrated that MSCs repair livers injured by disease mainly by differentiating into hepatocytes and modulating the immune system. Hepatocyte transplantation improves injured liver function by functional hepatocyte replacement. In this study, we found that compared with MSC transplantation, 

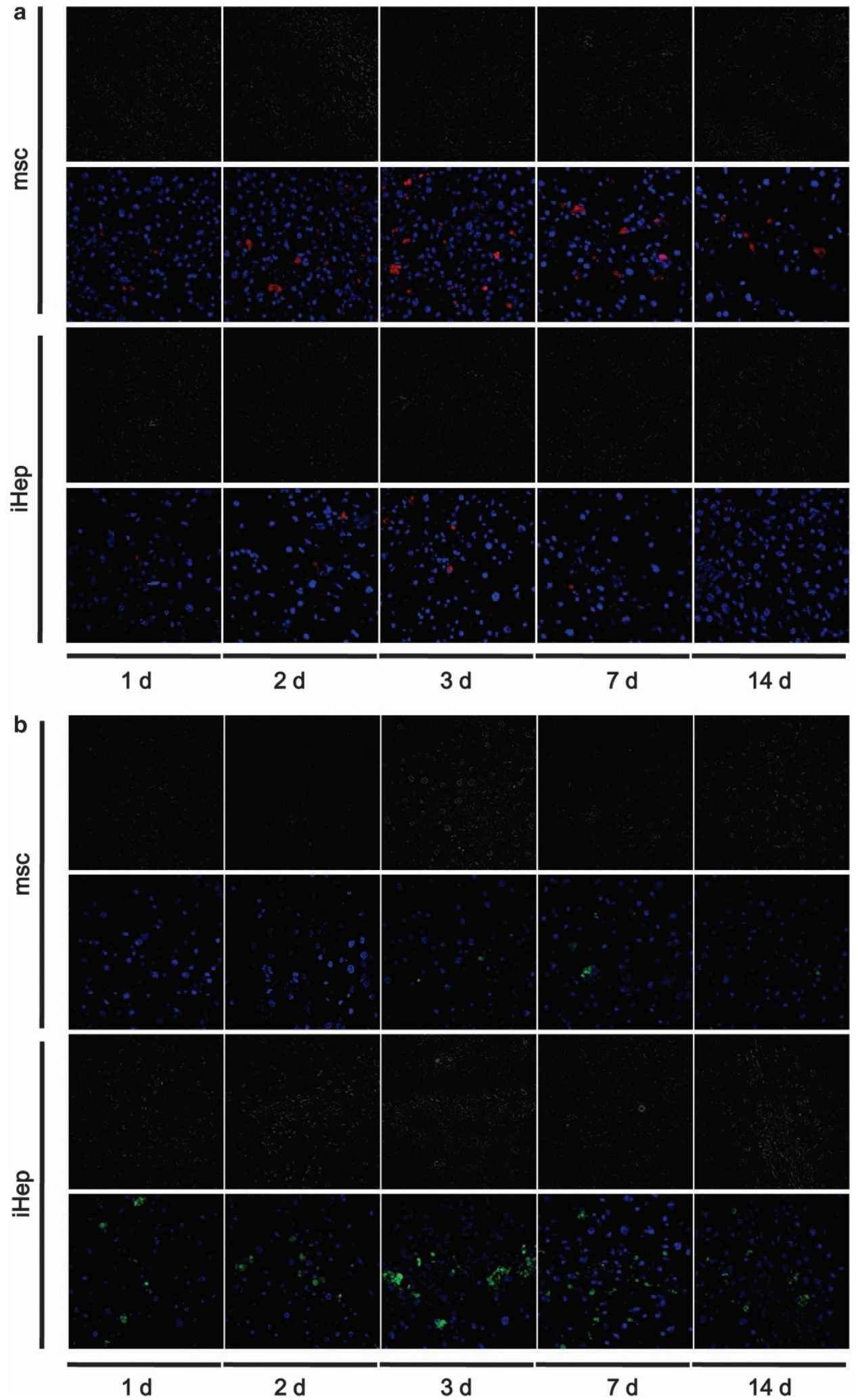

Figure 5 iHep cell tracing in the livers of injured mice. (a) Human-derived CD105 + cell analysis after iHep or hMSC cell transplantation. Red color represents the human CD105 antibody; blue color represents DAPI. (b) Human-derived albumin + cell analysis after iHep or hMSC cell transplantation. Green color represents the human albumin antibody; blue color represents DAPI 
seven-miRNA-mediated iHep transplantation improved liver function and increased serum albumin levels more efficiently ( 1 day and 7 days, respectively). More interestingly, transplanted iHeps not only supply functional hepatocytes but they also contain a small amount of CD105 + cells that are maintained on a stable level, indicating that seven-miRNAmediated iHeps possess stemness and could supply mature hepatocytes persistently.

\section{Materials and Methods \\ Cell culture and hepatic differentiation of mesenchymal stem cells. The isolation of human umbilical cord lining-derived mesenchymal stem cells was performed as previously described. ${ }^{6}$ Hepatic differentiation of hMSCs, which were infected with lentivirus for 6 days, was performed with a Hepatogenic Differentiation Kit (Cyagen Bioscience Inc., Guangzhou, China) as previously described. ${ }^{6}$}

Lentivirus vector construction and infection. The siRNAs miR-1246, miR-1290, miR-148a, miR-30a, miR-424 and miR-542-5p were packaged in the eGFP-GV273 vector using a lentiviral system by Genechem Co., Ltd., (Shanghai, China). HMSCs were plated at $6 \times 10^{3}$ cells/well in 24-well plates. After $24 \mathrm{~h}$, the hMSCs were infected with $10 \mu \mathrm{l}$ of $1 \times 10^{8} \mathrm{TU} / \mathrm{ml}(\mathrm{MOI}=100)$ miRNA-RNAi-Lev, miR-1290-RNAi-Lev, miR-148a-RNAi-Lev, miR-30a-RNAi-Lev, miR-424-RNAi-Lev, miR-542-5p-RNAi-Lev or a negative control RNA. Then, $10 \mu \mathrm{l}$ of polybrene was added to each well to reach a final concentration of $5 \mu \mathrm{g} / \mathrm{ml}$. After infection of the hMSCs, fluorescent protein expression in the infection and negative control groups was observed under an inverted fluorescence microscope at $24,48,72$ and $96 \mathrm{~h}$ to assess the infection efficiency.

Transfection of hMSCs with miRNA mimics. Mimics of miR-122, miR-148a, miR-424 and miR-542-5p were synthesized by GenePharma Co., Ltd. (Shanghai, China), and mimics of miR-1246, miR-1290 and miR-30a were synthesized from Ribobio Co., Ltd. (Guangzhou, China). As a negative control, we used a random non-targeting sequence mimic (mimic-nc) miRNA from GenePharma Co., Ltd. (Shanghai, China). Before transfection, the hMSCs were plated at $3 \times 10^{4}$ cells per well in 24-well plates. For each miRNA mimic transfection sample, $10 \mu \mathrm{l}$ of $20 \mu \mathrm{M}$ miRNA mimic was diluted in $50 \mu \mathrm{l}$ of OptiMEM I Reduced Serum Medium (Invitrogen Inc., Carlsbad, CA, USA). Then, $1 \mu \mathrm{l}$ of lipofectamine 2000 (Invitrogen Inc) was diluted in $50 \mu$ l of Reduced Serum Medium. After a 15-minute incubation, the two solutions were combined and added to each well containing cells and medium. For the seven-miRNA mimic mix transfection sample, $35 \mu \mathrm{l}$ (five $\mu \mathrm{l} / \mathrm{miRNA}^{*} 7$ miRNA) of $20 \mu \mathrm{M}$ the miRNA mimics was diluted in $175 \mu \mathrm{l}$ of Opti-MEM I Reduced Serum Medium. Then, $4 \mu \mathrm{l}$ of lipofectamine 2000 was diluted in $200 \mu \mathrm{l}$ of Reduced Serum Medium. After a 15-minute incubation, the two solutions were combined and added to each well containing cells and medium. The cells were harvested for mRNA and protein analysis at the indicated time points. We classify the cells mediated by the seven-miRNA combination as induced hepatocyte-like cells (iHep).

RNA isolation, cDNA synthesis and quantitative reverse transcription PCR (qRT-PCR). RNA isolation, CDNA synthesis and qRT-PCR were performed as previously described. ${ }^{6}$ Briefly, the total RNA was isolated with Trizol at the indicated time points (Invitrogen Inc). A total of $300 \mathrm{ng}$ of total RNA was used for CDNA synthesis with the PrimeScript RT Reagent Kit Perfect Real Time (TaKaRa Biotechnology Co. Ltd., Dalian, China). PCR amplification was performed with the SYBR Premix Ex Taq TM II (TaKaRa Biotechnology). For each sample, GAPDH expression was analyzed to normalize the target gene expression.

For miRNA analysis, cDNA was synthesized with the One Step PrimeScript miRNA cDNA Synthesis Kit Perfect Real Time (TaKaRa Biotechnology). Human U6B was used to normalize target miRNA expression. The primers for qRT-PCR are shown in Table 1. In all of the miRNA analyses, the Uni-miR qPCR primer was used as the reverse primer (TaKaRa Biotechnology). Relative changes in gene and miRNA expression were determined with the $2-\Delta \Delta C$ method.

Periodic acid-Schiff staining (PAS staining) and urea assay. Glycogen storage by the induced hepatocytes was analyzed with a PAS staining kit (Baso Diagnostics Inc., Zhuhai, China). The cells were fixed with PBS containing $4 \%$ paraformaldehyde, incubated for $10 \mathrm{~min}$ in $1 \%$ periodic acid, washed with distilled water and incubated with Schiff's reagent for $15 \mathrm{~min}$. After a 10-minute wash in tap water, the cells were visualized by light microscopy and images were acquired. For the urea assay, induced hepatocytes were cultured for $24 \mathrm{~h}$ in expansion medium in the presence or absence of $10 \mathrm{mmol} / / \mathrm{NH}_{4} \mathrm{Cl}$. Then, the supernatants were collected and the urea concentrations in the supernatants were measured by a BUN assay (Nanjing Jiancheng Bioengineering Institute, Jiangsu, China) according to the manufacturer's instructions. Expansion medium served as a negative control. Finally, the plates were read at a wavelength of $640 \mathrm{~nm}$ in an automatic microplate reader (BIO-RAD 680/Bio-Rad Laboratories, Hercules, CA, USA).

ICG and LDL uptake. For ICG uptake analysis, cardiogreen (Sigma-Aldrich Inc., St. Louis, MO, USA) was dissolved in sterile ddH2O to produce a fresh $50 \mathrm{mg} / \mathrm{ml}$ stock solution that was then further diluted in DMEM to a final concentration of $1 \mathrm{mg} / \mathrm{ml}$. After a 30-minute incubation and a 10-minute water wash, the cells were visualized by light microscopy. The LDL uptake ability of the

Table 1 Primers for the target genes for qRT-PCR analysis

\begin{tabular}{|c|c|c|c|}
\hline Target gene & Primer $\left(5^{\prime} \rightarrow 3^{\prime}\right)$ & Target gene & Primer $\left(5^{\prime} \rightarrow 3^{\prime}\right)$ \\
\hline CK-18 & $\begin{array}{l}\text { F: CCCTGCTGAACATCAAGGTCAA } \\
\text { R: GCTGTCCAAGGCATCACCAA }\end{array}$ & EpCAM & $\begin{array}{l}\text { F: GAATGGCAAAGTATGAGAAGGCTGA } \\
\text { R: TCCCACGCACACACATTTGTAA }\end{array}$ \\
\hline HNF4A & $\begin{array}{l}\text { F: AGCTGCAGATCGATGACAATGAG } \\
\text { R: CATACTGGCGGTCGTTGATGTAG }\end{array}$ & G6P & $\begin{array}{l}\text { F: CCTTTGGGTAGCTGTGATTGGA } \\
\text { R: GGCACGGAAGTGTTGCTGTAGTAG }\end{array}$ \\
\hline ALB & $\begin{array}{l}\text { F: ACTGCATTGCCGAAGTGGA } \\
\text { R: GCAGCACGACAGAGTAATCAGGA }\end{array}$ & AFP & $\begin{array}{l}\text { F: AGAAACCCACTGGAGATGAACAGTC } \\
\text { R: GGCTGCAGCAGTCTGAATGTC }\end{array}$ \\
\hline GAPDH & $\begin{array}{l}\text { F: GCACCGTCAAGGCTGAGAAC } \\
\text { R: TGGTGAAGACGCCAGTGGA }\end{array}$ & CK7 & $\begin{array}{l}\text { F: GCTGAGGCTGAAGCCTGGTA } \\
\text { R: CATCTCTGAAATCTCATTCCGGGTA }\end{array}$ \\
\hline CYP3A4 & $\begin{array}{l}\text { F: AAGTCGCCTCGAAGATACACA } \\
\text { R: AAGGAGAGAACACTGCTCGTG }\end{array}$ & PDX1 & $\begin{array}{l}\text { F: ACTCCACCTTGGGACCTGTTTAGA } \\
\text { R: CGAGTAAGAATGGCTTTATGGCAGA }\end{array}$ \\
\hline TF & $\begin{array}{l}\text { F: CCTCCTACCTTGATTGCATCAG } \\
\text { R: TTTTGACCCATAGAACTCTGCC }\end{array}$ & $\begin{array}{l}\operatorname{miR}-148 a \\
\text { miR-1246 }\end{array}$ & $\begin{array}{l}\text { TCAGTGCACTACAGAACTTTGT } \\
\text { AATGGATTTTTGGAGCAGG }\end{array}$ \\
\hline $\begin{array}{l}\text { miR-122 } \\
\text { miR-30a } \\
\text { miR-542-5p }\end{array}$ & $\begin{array}{l}\text { TGGAGTGTGACAATGGTGTTTG } \\
\text { TGTAAACATCCTCGACTGGAAG } \\
\text { TCGGGGATCATCATGTCACGAGA }\end{array}$ & $\begin{array}{l}\operatorname{miR}-1290 \\
\mathrm{miR}-424\end{array}$ & $\begin{array}{l}\text { TGGATTTTTGGATCAGGGA } \\
\text { CTTCCCCCCAGTAATCTTCATC }\end{array}$ \\
\hline
\end{tabular}


induced hepatocytes was assessed by fluorescence microscopy after incubation of the cells with $10 \mathrm{mg} / \mathrm{ml}$ acetylated LDL labeled with 1, 19-dioctadecyl-3,3,39, 39-tetramethylindo-carbocyanine perchlorate (Dil-Ac-LDL) (Yiyuan Biotechnologies, Guangzhou, China) for 4 hours at $37^{\circ} \mathrm{C}$. The medium was removed and the cells were washed with probe-free medium and visualized by fluorescence microscopy.

Western blot. The cell lysates from the induced hepatocytes were extracted with RIPA lysis buffer (Beyotime Inc., Shanghai, China). The samples were resolved in a $10 \%$ SDS-PAGE gel and transferred to a PVDF membrane (Millipore Corporation, Billerica, MA, USA) using the semi-dry transfer method. After blocking in $10 \%$ non-fat dried milk in TBST for $2 \mathrm{~h}$, the blots were incubated with primary antibody at $4{ }^{\circ} \mathrm{C}$ overnight. After washing with TBST, the blots were incubated with a horseradish peroxidase-conjugated secondary antibody (Santa Cruz Biotechnology, Santa Cruz, CA, USA, diluted 1:2000) at room temperature for $1 \mathrm{~h}$. The blots were visualized by Femto (Pierce, Rockford, IL, USA) following the manufacturer's instructions using the following primary antibodies: human anti-ALB (Santa Cruz), human anti-HNF4A (Santa Cruz Biotechnology) and human anti-CYP3A4 (Santa Cruz Biotechnology).

Cell transplantation and labeling. Eight-week-old female BALB/c nude mice from the animal center of The Fourth Military Medical University received intraperitoneal injections of $10 \% \mathrm{CCl} 4$ that were diluted in corn oil solution $(2 \mathrm{ml} / \mathrm{kg})$ three times a week for 4 weeks. The mice with $\mathrm{CCl} 4$-induced liver injury were divided randomly into three groups: Group A: treated with saline $(n=15)$; Group B: treated with human negative cell, which was human blood-derived CD34-negative non-adherent cells ( $n=15)$; Group C: injected with human MSCs $(n=15)$; Group D: injected with human induced hepatocyte derived from MSCs $(n=15)$. Each mouse received $1 \times 10^{6}$ cells via the tail vein. The mouse sera and liver tissues were harvested at 1, 2, 3, 7 and 14 days. Mouse serum parameters, including albumin (ALB), alanine aminotransferase (ALT) and aspartate aminotransferase (AST), were analyzed with an automatic chemistry analyzer Au560 (Olympus, Tokyo, Japan) in a clinical laboratory. Fresh liver segments were prepared for H\&E staining and immunofluorescence.

Immunofluorescence. Fresh liver segments were fixed with 4\% paraformaldehyde. After blocking with phosphate-buffered saline containing $1 \%$ BSA and $0.2 \%$ Triton X-100, the cells were incubated with anti-human-albumin antibody (Santa Cruz Biotechnology) or anti-human CD105-PE (eBioscience Inc., San Diego, CA, USA) at $4^{\circ} \mathrm{C}$ overnight and then incubated with a secondary antibody that was labeled with Alexa Fluor 488 (Invitrogen Inc.) at room temperature for $1 \mathrm{~h}$.

Statistical analysis. The data are expressed as the mean \pm standard deviation. To identify significant differences, a one-way analysis of variance was performed and the least significant difference $t$-test was used to analyze the differences between the groups. A $P$-value $<0.05$ was considered significant.

\section{Conflict of Interest}

The authors declare no conflict of interest.

Acknowledgements. This work was supported by The National Natural Science Foundation of China (No. 81070326) and The National Science and Technology Projects of China (2014ZX09508002). We thank Yongzhan Nie, Zheng Chen and Zuhong Tian for their technical guidance during this study.

1. Huang $P$, He Z, Ji S, Sun H, Xiang D, Liu $C$ et al. Induction of functional hepatocyte-like cells from mouse fibroblasts by defined factors. Nature 2011; 475: 386-389.

2. Sekiya S, Suzuki A. Direct conversion of mouse fibroblasts to hepatocyte-like cells by defined factors. Nature 2011; 475: 390-393.

3. Shenoy A, Blelloch R. microRNA induced transdifferentiation. F1000 Biol Rep 2012; 4: 3 .
4. Yoo AS, Sun AX, Li L, Shcheglovitov A, Portmann T, Li Y et al. MicroRNA-mediated conversion of human fibroblasts to neurons. Nature 2011; 476: 228-231.

5. Jayawardena TM, Egemnazarov B, Finch EA, Zhang L, Payne JA, Pandya K et al. MicroRNA-mediated in vitro and in vivo direct reprogramming of cardiac fibroblasts to cardiomyocytes. Circ Res 2012; 110: 1465-1473.

6. Cui L, Zhou X, Li J, Wang L, Wang J, Li Q et al. Dynamic microRNA profiles of hepatic differentiated human umbilical cord lining-derived mesenchymal stem cells. PLoS One 2012; 7: e44737.

7. Hand NJ, Master ZR, Le Lay J, Friedman JR. Hepatic function is preserved in the absence of mature microRNAs. Hepatology 2009; 49: 618-626.

8. Song G, Sharma AD, Roll GR, Ng R, Lee AY, Blelloch RH et al. MicroRNAs control hepatocyte proliferation during liver regeneration. Hepatology 2010; 51: 1735-1743.

9. Chen H, Sun Y, Dong R, Yang S, Pan C, Xiang D et al. Mir-34a is upregulated during liver regeneration in rats and is associated with the suppression of hepatocyte proliferation. PLOS One 2011; 6: e20238.

10. Castro RE, Ferreira DM, Zhang X, Borralho PM, Sarver AL, Zeng $Y$ et al. Identification of microRNAs during rat liver regeneration after partial hepatectomy and modulation by ursodeoxycholic acid. Am J Physiol Gastrointest Liver Physiol 2010; 299: G887-G897.

11. Zhang Y, Liao JM, Zeng SX, Lu H. p53 downregulates Down syndrome-associated DYRK1A through miR-1246. EMBO Rep 2011; 12: 811-817.

12. Gillen $\mathrm{AE}$, Gosalia N, Leir $\mathrm{SH}$, Harris A. MicroRNA regulation of expression of the cystic fibrosis transmembrane conductance regulator gene. Biochem J 2011; 438: 25-32.

13. Pigati L, Yaddanapudi SC, lyengar R, Kim DJ, Hearn SA, Danforth D et al. Selective release of microRNA species from normal and malignant mammary epithelial cells. PLOS One 2010; 5: e13515.

14. Wu J, Ji X, Zhu L, Jiang Q, Wen Z, Xu S et al. Up-regulation of microRNA-1290 impairs cytokinesis and affects the reprogramming of colon cancer cells. Cancer Lett 2013; 329: 155-163.

15. Kumarswamy R, Mudduluru G, Ceppi P, Muppala S, Kozlowski M, Niklinski J et al. MicroRNA-30a inhibits epithelial-to-mesenchymal transition by targeting Snai1 and is downregulated in non-small cell lung cancer. Int J Cancer 2012; 130: 2044-2053.

16. Hand NJ, Master ZR, Eauclaire SF, Weinblatt DE, Matthews RP, Friedman JR. The microRNA-30 family is required for vertebrate hepatobiliary development. Gastroenterology 2009; 136: 1081-1090.

17. Farid WR, Pan Q, van der Meer AJ, de Ruiter PE, Ramakrishnaiah V, de Jonge J et al. Hepatocyte-derived microRNAs as serum biomarkers of hepatic injury and rejection after liver transplantation. Liver Transp/ 2012; 18: 290-297.

18. Rosa A, Ballarino M, Sorrentino A, Sthandier O, De Angelis FG, Marchioni M et al. The interplay between the master transcription factor PU.1 and miR-424 regulates human monocyte/macrophage differentiation. Proc Natl Acad Sci USA 2007; 104: 19849-19854.

19. Lulla RR, Costa FF, Bischof JM, Chou PM, de F Bonaldo M, Vanin EF et al. Identification of differentially expressed microRNAs in osteosarcoma. Sarcoma 20112011732690.

20. Bray I, Tivnan A, Bryan K, Foley NH, Watters KM, Tracey L et al. MicroRNA-542-5p as a novel tumor suppressor in neuroblastoma. Cancer Lett 2011; 303: 56-64.

21. Faraonio R, Salerno P, Passaro F, Sedia $C$, laccio A, Bellelli $R$ et al. A set of miRNAs participates in the cellular senescence program in human diploid fibroblasts. Cell Death Differ 2012; 19: 713-721.

22. Jopling C. Liver-specific microRNA-122: biogenesis and function. RNA Biol 2012; 9: 137-142.

23. Doddapaneni R, Chawla YK, Das A, Kaur J, Ghosh S, Chakraborti A. Overexpression of microRNA-122 enhances in vitro hepatic differentiation of fetal liver-derived stem/progenitor cells. J Cell Biochem 2013; 114: 1575-1583.

24. Lee KD, Kuo TK, Whang-Peng J, Chung YF, Lin CT, Chou SH et al. In vitro hepatic differentiation of human mesenchymal stem cells. Hepatology 2004; 40: 1275-1284.

25. lacob R, Rudrich U, Rothe M, Kirsch S, Maasoumy B, Narain N et al. Induction of a mature hepatocyte phenotype in adult liver derived progenitor cells by ectopic expression of transcription factors. Stem Cell Res 2011; 6: 251-261.

26. Takayama K, Inamura M, Kawabata K, Sugawara M, Kikuchi K, Higuchi M et al. Generation of metabolically functioning hepatocytes from human pluripotent stem cells by FOXA2 and HNF1alpha transduction. J Hepatol 2012; 57: 628-636.

(c) (i) $(\theta)$ Cell Death and Disease is an open-access journal published by Nature Publishing Group. This work is licensed under a Creative Commons Attribution-NonCommercialNoDerivs 3.0 Unported License. To view a copy of this license, visit http://creativecommons.org/licenses/by-nc-nd/3.0/ 